PROGRAMA DE INICIAÇÃO CIENTÍFICA

\author{
ANA CAROLINA MOTA DE FARIA
}

LEVANTAMENTO DA MASTOFAUNA TERRESTRE DE MÉDIO E GRANDE PORTE EM REMANESCENTES FLORESTAIS DE CERRADO DA MICROBACIA DO CÓRREGO URUBU, DISTRITO FEDERAL 


\section{Unले $U B$ \\ Centro Unıversıtárıo de Brasílıa}

ANA CAROLINA MOTA DE FARIA

LEVANTAMENTO DA MASTOFAUNA TERRESTRE DE MÉDIO E GRANDE PORTE EM REMANESCENTES FLORESTAIS DE CERRADO DA MICROBACIA DO CÓRREGO URUBU, DISTRITO FEDERAL

\footnotetext{
Relatório final de pesquisa de Iniciação Científica apresentado à Assessoria de Pós-Graduação e Pesquisa pela Faculdade de Ciências da

Educação e da Saúde - FACES.

Orientação: Carlos Alberto da Cruz Júnior
} 


\title{
LEVANTAMENTO DA MASTOFAUNA TERRESTRE DE MÉDIO E GRANDE PORTE EM REMANESCENTES FLORESTAIS DE CERRADO DA MICROBACIA DO CÓRREGO URUBU, DISTRITO FEDERAL
}

\author{
Ana Carolina Mota de Faria - UniCEUB, PIBIC-CNPq, aluna bolsista \\ anacarolina.mota.faria@gmail.com
}

Carlos Alberto da Cruz Júnior - UniCEUB, professor orientador carlos.junior@uniceub.com

Fábio Hudson Souza Soares - UnB, colaborador fabiohudsoares@gmail.com

Bernardo Ramos Simões Corrêa - UnB, colaborador bernersc@hotmail.com

\section{Vitor Ramos Simões Corrêa - UPIS, colaborador} quintadelfim@gmail.com

A conservação dos fragmentos de vegetação nativa associados ao conhecimento da distribuição e da localização da mastofauna é indispensável para a sua preservação. O desenvolvimento de estudos com levantamentos de espécies utilizando armadilhas fotográficas como ferramenta de coleta de dados é fundamental para subsidiar propostas de manejo e conservação dos mamíferos de médio e grande porte, bem como para o controle dos ecossistemas. O presente estudo foi realizado como o objetivo de inventariar e avaliar a abundância e a diversidade de mamíferos terrestres presentes na Estação Experimental de Agroecologia - Chácara Delfim, localizado na Microbacia do Córrego Urubu do Distrito Federal. A microbacia do Córrego Urubu é uma área considerada de proteção ambiental, caracterizada como um fragmento de cerrado, que se situa em área de recarga da sub-bacia Norte do Lago Paranoá, que concentra importantes nascentes da Área de Proteção Ambiental - APA do Planalto Central, e que vê sua área diminuída com o avanço da urbanização promovida inadvertidamente pela especulação imobiliária. Trata-se um dos poucos fragmentos de cerrado existentes na região e ainda margeado por propriedades rurais. A área de estudo está localizada em um fragmento de mata de galeria e savana de aproximadamente 10 hectares, onde ocorre um vale que apresenta bordas de mudança brusca de declividade evidente. O entorno do fragmento apresenta características urbanas bem definidas, como condomínios residências de alto padrão (Setor habitacional Taquari etapa I) e um bairro de baixa renda (Varjão), apesar de estar localizado em área rural. O estudo ocorreu durante nove meses consecutivos, entre novembro de 2016 e agosto de 2017, com a utilização de duas armadilhas fotográficas e a busca de rastros, pegadas e vestígios de fezes. Durante o trabalho, foram observadas oito espécies de mamíferos terrestres, incluindo duas espécies exóticas que ocorrem na região. Apesar de o estudo ter sido conduzido em um fragmento de cerrado obtiveram-se registros de dois mamíferos de grande porte como 
- Mazama gouazoupira e Chrysocyon brachyurus. As armadilhas fotográficas apresentaram um esforço amostral de 458 armadilhas-dias e exposição de 10.992 horas de amostragem. As espécies que apresentaram maior número de registros foram aquelas que mais se adaptam a ambientes modificados.

Palavras-Chave: Armadilhas-fotográficas. Conservação. Câmeras-Trap. Lobo. 


\section{Agradecimentos}

Agradeço à todos que acreditaram no nosso trabalho e que colaboraram de maneira direta ou indireta para o enriquecimento do estudo, em especial, para todas as pessoas que integram a Chácara Delfim, que sempre nos receberam com muito carinho.

Meus sinceros agradecimentos ao Coordenador Carlos Alberto da Cruz Júnior, por toda a confiança, comprometimento e por ter me orientado até a conclusão desse projeto.

Aos colegas Bernardo Ramos, Fábio Hudson, Vitor Ramos, Ana Luiza e Luísa Caroline por toda a colaboração prestada durante a realização desse estudo.

Ao $\mathrm{CNPq}$ pela bolsa concedida para a realização do projeto. Agradeço também ao Programa de assessoria de Pós-Graduação e Pesquisa do UniCEUB que sempre mostraram apoio no desenvolvimento do nosso trabalho.

Meus agradecimentos especiais aos meus pais Cleunice Mota e Welington Faria, e a toda minha família e meus amigos que sempre me apoiaram e estiveram ao meu lado durante todo esse tempo. 


\section{Sumário}

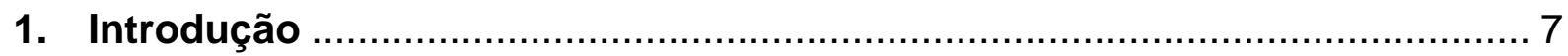

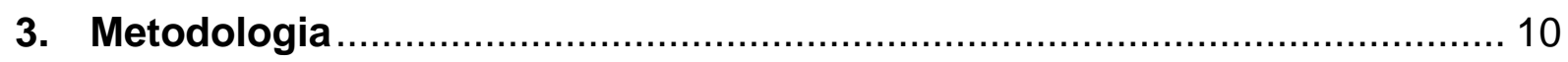

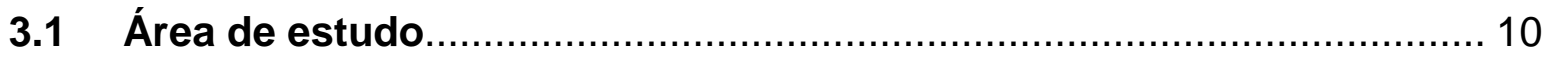

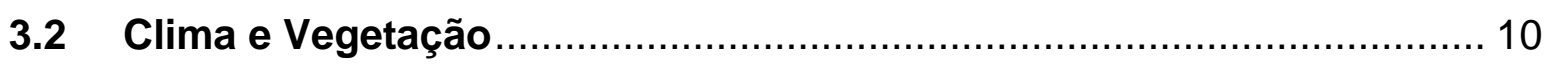

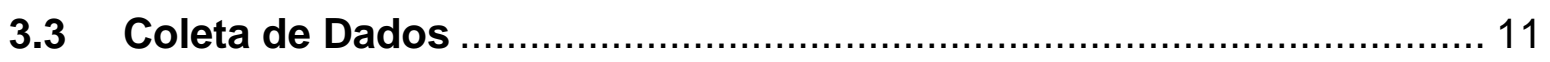

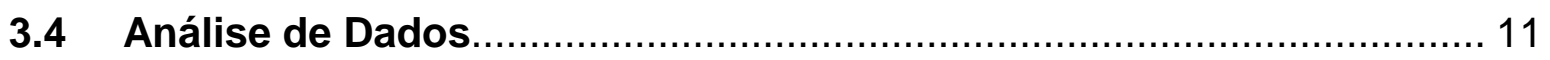

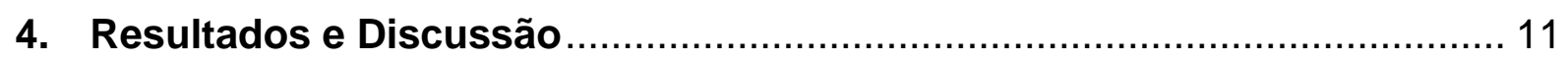

4.1 Composição e Riqueza ................................................................... 11

Tabela 1. Taxa (ordem, família e espécie) de acordo com o ICMBio, nome comum, categoria ( $\mathrm{MP}=$ Menos preocupante, $\mathrm{QA}=$ Quase ameaça) na lista vermelha internacional (IUCN, 2017) e o número de registros de cada espécie localizadas na Estação Experimental de Agroecologia - Chácara Delfim, localizado na Microbacia do Córrego Urubu do Distrito Federal. ...................... 12

Figura 1. Ocorrência das espécies de mamíferos de médio e grande porte registradas durante o levantamento realizado entre os meses de novembro de 2016 e julho de 2017 com armadilhas fotográficas representadas por gráficos. 13

\subsection{Fitofisionomias e Uso do habitat ..................................................... 14}

Figura 2. Análise da fitofisionomia da Estação Experimental de Agroecologia comparadas ao número de espécies registradas por cada área amostrada...... 14

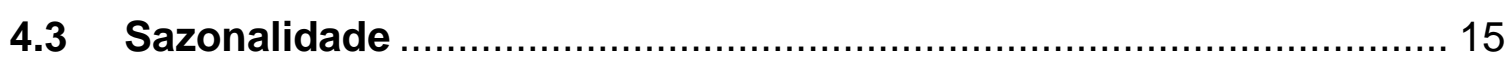

Tabela 2. Ocorrências registradas das espécies de mamíferos de médio e grande porte durante os meses de Novembro de 2016 e Julho de 2017 na Estação Experimental de Agroecologia - Chácara Delfim, localizado na Microbacia do Córrego Urubu do Distrito Federal......................................... 15

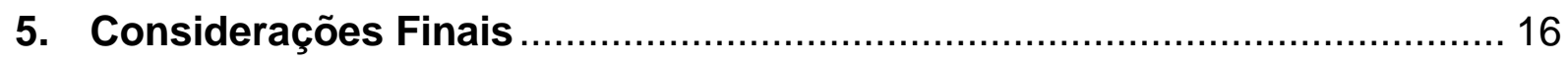

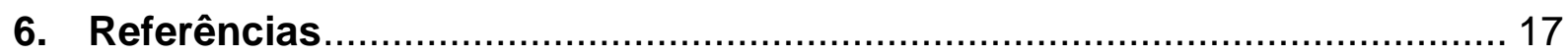




\section{Introdução}

Um dos maiores desafios para a humanidade é a conservação da biodiversidade, devido ao elevado nível de fragmentação gerado nos ecossistemas naturais para o estabelecimento das grandes cidades. No Distrito Federal, o processo de urbanização acelerada e desordenada ocorre como em diversas outras capitais, onde áreas urbanas formadas por bairros e assentamentos precários substituem zonas rurais com áreas de preservação ambiental e mananciais, consequentemente tornando os ecossistemas altamente vulneráveis.

O interesse no estudo das consequências da fragmentação florestal sobre a conservação da biodiversidade tem aumentado significativamente nos últimos anos devido à constatação de que a maior parte da biodiversidade se encontra hoje localizada em pequenos fragmentos florestais, pouco estudados e historicamente marginalizados pelas iniciativas conservacionistas. A fragmentação introduz uma série de novos fatores na história evolutiva de populações naturais de plantas e animais. Essas mudanças afetam de forma diferenciada os parâmetros demográficos de mortalidade e natalidade de diferentes espécies, portanto, a estrutura e dinâmica de ecossistemas.

Nos últimos 35 anos o bioma cerrado foi um dos ecossistemas com maior interferência antrópica (AQUINO \& MIRANDA, 2008). No Distrito Federal, a Microbacia do Córrego Urubu vem sofrendo fragmentação decorrente de mudanças no uso do solo, através da implantação do Setor Habitacional Taquari Etapa 1 e 2, ficando ilhada e com pequenas dimensões, em meio a urbanização e a acelerada ação depredatória do homem.

Os remanescentes dos fragmentos florestais são importantes para a persistência de diversas espécies de mamíferos terrestres de médio e grande porte. Estes animais desempenham papel fundamental na manutenção da diversidade de uma área, sendo dispersores e predadores de sementes, predadores de plântulas e reguladores de níveis tróficos inferiores (PALOMARES et al., 1995; BECK-KING, 1999; HENRY, 1999). Devido à destruição de seus hábitats naturais e suas características ecológicas, como baixa densidade populacional e ocupação de grandes territórios, muitas espécies de mamíferos de médio e grande porte se encontram ameaçadas de extinção (MARINHO-FILHO et al., 2002). 
Os fragmentos impõem restrições à permanência de espécies devido à limitação da área de vida, efeitos de borda, escassez de recursos e restrição à dispersão provocada pela matriz circundante (CÂNDIDO JR., 2000; FAHRIG, 2003; COSTA et al., 2005), aumentando o endocruzamento e podendo levar as populações à extinção. Em situações de fragmentação dos ambientes naturais, mamíferos de médio e grande porte tendem a enfrentar um problema adicional, que é a forte pressão da caça ilegal, favorecida em áreas restritas como fragmentos (CHIARELLO, 1999; CULLEN-JR. et al., 2000; CULLEN-JR. et al., 2001; PERES, 2001; ROCHAMENDES et al., 2005). Assim, a sobrevivência dessas espécies, que são preferidas pelos caçadores, tornase ainda mais ameaçada.

Os pré-requisitos indispensáveis para o desenvolvimento de ações conservacionistas, principalmente no que se refere à sua biodiversidade, estão na dependência do conhecimento básico das espécies e sua distribuição espacial (KASPER et al. 2007) e de acordo com Secades et al (2014) o monitoramento constitui-se parte essencial da conservação uma vez que permite identificar problemas, desenvolver soluções e avaliar a efetividade de ações e progressos.

O presente projeto de pesquisa tem por objetivo realizar um levantamento das espécies de mamíferos terrestres de médio e grande porte no fragmento de cerrado inserido na Microbacia do Córrego do Urubu, localizada na região administrativa do Lago Norte, Distrito Federal, Brasil.

\section{Fundamentação Teórica}

Os mamíferos com ocorrência no Cerrado totalizam cerca de 195 espécies, sendo que 18 delas são endêmicas e 17 estão incluídas na lista nacional das espécies da fauna brasileira ameaçadas de extinção (MMA, 2003). A redução e o isolamento de populações devido à fragmentação de habitats e o isolamento dos remanescentes florestais podem levar a diminuição da diversidade genética e aumento da chance de extinção estocástica dessas populações (WILCOX, 1985; FUTUYMA, 1997). Além disso, também podem ser observadas modificações no habitat remanescente por meio de alterações causadas pela influência das áreas alteradas do entorno (LAURANCE, 2008).

O conhecimento da biologia dos mamíferos de maior porte tem evidenciado sua importância em uma série de processos nos ecossistemas florestais. Espécies frugívoras e/ou herbívoras, como os grandes roedores, veados, porcos e anta, têm 
papel importante na dispersão de sementes e na predação de sementes e plântulas, e sua ausência tem profundas repercussões na floresta, afetando a composição, diminuindo a diversidade e alterando a regeneração da vegetação (TERBORGH, 1988; DIRZO, 1990; CARRILLO et al. 2000; De MATIA et al. 2004). Por outro lado, os mamíferos carnívoros, principalmente os predadores de topo de cadeia como as onças, parecem ser importantes para o controle "top-down" (através da predação de cima para baixo nas teias alimentares) das populações dos mamíferos herbívoros, e de mamíferos de médio porte generalistas, chamados de meso-predadores (TERBORGH et al. 2001). Em locais onde os carnívoros predadores de topo foram localmente extintos é observada uma diminuição na riqueza de espécies de aves e outros pequenos vertebrados, associada a um aumento na predação por mesopredadores (FONSECA,1990; PALOMARES et al. 1995; CROOKS,1999).

Apesar da sensibilidade e da importância ecológicas dos mamíferos de maior porte, poucos estudos abordaram os efeitos da fragmentação ou da estrutura da paisagem sobre estes animais devido às dificuldades impostas pelas baixas densidades e hábitos noturnos e discretos da maioria das espécies. Em um dos poucos estudos sobre os efeitos da fragmentação em mamíferos de maior porte da Mata Atlântica, Chiarello (1999) encontrou que a estrutura desta comunidade é fortemente relacionada ao tamanho dos remanescentes. Comunidades de mamíferos em grandes reservas (a partir de 20.000 ha) apresentam maior complexidade, com maior riqueza de espécies, abrigando predadores de topo, grandes frugívoros terrestre e tamanduás. Já nas pequenas reservas (200 ha) encontra-se uma menor complexidade e menor número de espécies de mamíferos de maior porte, com uma redução de espécies topo de cadeia e proliferação de algumas espécies associadas à vegetação secundária (CHIARELLO, 1999).

Compreender as mudanças nas populações e comunidades entre diferentes ambientes e a dinâmica e as relações entre mosaicos de paisagens são prioridades para o planejamento da conservação das florestas tropicais (CHAZDON et al. 2009; GARDNER et al. 2009).

O uso de armadilhas fotográficas são bastante empregadas em levantamentos qualitativos (SANTOS-FILHO, 2002; SILVEIRA et al., 2003; TROLLE, 2003 a; b; ALVES, 2005; SRBEK-ARAUJO, 2005) pois além de auxiliarem a identificação taxonômica, podem ser de grande valia para facilitar ações em projetos de pesquisa, uma vez que fornecem horários das atividades de espécies da fauna e podem também 
acrescentar dados importantes ao conhecimento da história natural das mesmas (NASCIMENTO et al., 2004).

A mastofauna apresenta em sua maioria hábitos crepusculares e noturnos, dificultando a sua visualização por parte da comunidade local, dessa forma o uso de câmeras trap aumenta as chances de captura de imagens desses animais.

Outra forma eficiente é a identificação por rastros e vestígios, geralmente deixados durante a realização de suas atividades sendo a forma mais acessível e concreta de inventariar a mastofauna pela pelas populações locais. A partir deste pressuposto, Becker e Dalponte (2013) indicam que pegadas, restos de repastos, abrigos, tocas, ninhos, fezes, rastros e outros sinais são reveladores da presença e das atividades de mamíferos e são facilmente encontrados.

Aliada à dimensão espacial, a dimensão temporal de estudos ecológicos também precisa ser considerada, uma vez que os sistemas ecológicos estão em constante mudança ao longo do tempo. O monitoramento em longo prazo se torna, dessa forma, essencial para conhecermos as taxas e direções de mudanças nesses sistemas, permitindo diferenciar comportamentos naturais daqueles associados à ação humana.

\section{Metodologia}

\section{1 Área de estudo}

A área de estudo está localizada em um fragmento de mata de galeria e savana de aproximadamente 10 hectares da Estação Experimental de Agroecologia do Centro Universitário de Brasília, localizada na Chácara Delfim, Córrego do Urubu (15ํㅜ $42^{\prime}$ 0,05”S 47ำ 51'27,8”O), pertencente a região administrativa do Lago Norte - Distrito Federal. No fragmento ocorre um vale que apresenta bordas de mudança brusca de declividade evidente.

\subsection{Clima e Vegetação}

O entorno do fragmento apresenta características urbanas bem definidas, como Condomínios Residenciais de Alto Padrão (Setor Habitacional Taquari Etapa I) e um bairro de baixa renda (Varjão), apesar de estar localizado em área rural. Quanto ao zoneamento da área de estudo, encontra-se na unidade hidrográfica Santa Maria/Torto (bacia hidrográfica do lago Paranoá) e está inserida em duas importantes Áreas de Proteção Ambiental (APA) do Planalto Central e do lago Paranoá. 
O clima da região é o tropical com estação seca (do tipo Aw na classificação climática de Köppen-Geiger), com temperaturas médias mensais sempre superiores a $18{ }^{\circ} \mathrm{C}$ e índice pluviométrico em torno de 1.540 milímetros $(\mathrm{mm})$ anuais, concentrados entre os meses de outubro e abril.

\subsection{Coleta de Dados}

O fragmento de cerrado foi inicialmente georreferenciado e divido em quadrantes para a escolha de 10 pontos no interior do fragmento e marcados com o GPS (Garmin) para o controle das amostragens. Inicialmente 2 pontos foram sorteados e para cada ponto uma armadilha fotográficas (câmeras Trap), no qual foram fixadas em troncos de árvores a uma altura aproximada de $45 \mathrm{~cm}$ do solo.

As câmeras foram instaladas em locais que haviam rastros de animais e próximos a cursos d'águas para otimizar a captura das imagens. As armadilhas ficaram ativas por 15 dias em cada ponto e 24 horas por dia, sendo acionadas por sistema com sensor de calor e movimento.

\subsection{Análise de Dados}

Foram configuradas para gravar a data e hora na fotografia, registrando uma imagem a cada um minuto. Visando reduzir contagens sucessivas de um mesmo indivíduo, foram considerados registros efetivos, imagens fotografadas em um intervalo de cinco minutos. As imagens foram coletadas das câmeras a cada 7 dias e transferidas para análise com o programa de computação CAPTURE. Nessas datas foram realizadas as manutenções que se fizeram necessárias nos equipamentos como troca de pilhas. Após 15 dias outros 2 pontos foram sorteados e assim sucessivamente entre novembro de 2016 e julho de 2017. Além disso, nos últimos meses, foram utilizadas iscas, como pedaços de frutas que visavam capturar imagens dos mamíferos terrestres do fragmento. Durante o estudo, foram utilizadas também as metodologias indiretas (rastros e pegadas) e observações diretas (visual e auditiva) em todas as atividades de campo.

\section{Resultados e Discussão}

\subsection{Composição e Riqueza}

Foram obtidos o registro de oito espécies de mamíferos de médio e grande porte que apresentaram entre si diferentes abundâncias, do qual somaram um total de 127 mamíferos capturados, incluindo o registro de duas espécies exóticas, tais como 
Canis familiaris e Felis catus que ocorrem com frequência na região como mostra a figura 1.

Com o uso das armadilhas fotográficas, foram identificados também a presença de duas aves tipicamente encontradas no cerrado brasileiro sendo elas, Rhynchotus rufescens e Caracara plancus, durante o período de estudo na Estação Experimental de Agroecologia na Microbacia do Córrego Urubu do Distrito Federal.

Além disso, foi observado que os indivíduos que mais se adaptam à ambientes modificados foram os que obtiveram o maior número de registros através do uso de câmeras-trap (Tabela 1). As armadilhas fotográficas apresentaram um esforço amostral de 458 armadilhas-dias e exposição de 10.992 horas de amostragem durante 0 experimento.

Tabela 1. Taxa (ordem, família e espécie) de acordo com o ICMBio, nome comum, categoria $(\mathrm{MP}=$ Menos preocupante, $\mathrm{QA}=$ Quase ameaça) na lista vermelha internacional (IUCN, 2017) e o número de registros de cada espécie localizadas na Estação Experimental de Agroecologia - Chácara Delfim, localizado na Microbacia do Córrego Urubu do Distrito Federal.

\begin{tabular}{|c|c|c|c|}
\hline Taxa & Nome Comum & IUCN & Registros \\
\hline \multicolumn{4}{|l|}{ Ordem Didelphimorphia } \\
\hline $\begin{array}{l}\text { Família Didelphidae } \\
\text { Didelphis marsupialis }\end{array}$ & Gambá, Saruê & MP & 43 \\
\hline \multicolumn{4}{|l|}{ Ordem Primates } \\
\hline $\begin{array}{l}\text { Família Callitrichidae } \\
\text { Callithrix penicillata }\end{array}$ & Sagüi, mico-estrela & MP & 31 \\
\hline \multicolumn{4}{|l|}{ Ordem Cingulata } \\
\hline \multicolumn{4}{|l|}{ Família Dasypodidae } \\
\hline Cabassous unicinctus & Tatu-de-rabo-mole & MP & 2 \\
\hline Dasypus septemcinctus & Tatu-mulita, Tatuí & MP & 7 \\
\hline \multicolumn{4}{|l|}{ Ordem Lagomorpha } \\
\hline \multicolumn{4}{|l|}{ Família Leporidae } \\
\hline Sylvilagus brasiliensis & Coelho-do-mato, Candiba & MP & 10 \\
\hline \multicolumn{4}{|l|}{ Ordem Artiodactyl } \\
\hline \multicolumn{4}{|l|}{ Família Cervidae } \\
\hline Mazama gouazoubira & Veado Campeiro & MP & 1 \\
\hline \multicolumn{4}{|l|}{ Ordem Carnivora } \\
\hline \multicolumn{4}{|l|}{ Família Canidae } \\
\hline Chrysocyon brachyurus & Lobo-guará & QA & 1 \\
\hline
\end{tabular}

Apesar dos mamíferos registrados pelas armadilhas fotográficas não apresentarem um grau preocupante de ameaça pela lista vermelha da IUCN (IUCN, 2017), os índices de similaridades entre as espécies exóticas, incluindo os cães e 
gatos, apresentam uma grande ameaça aos mamíferos silvestres da área de conservação, uma vez que estes são competidores diretos por recursos alimentares e, além disso, têm a capacidade de predar animais silvestres e transmitir doenças. Assim como a não detecção de algumas espécies registradas pelo uso das armadilhas fotográficas representam baixas densidades populacionais (PARDINI et al. 2006) ou pouca mobilidade da área, associados à pressão da caça predatória, sendo raro a identificação de algum vestígio (LIMA, 2009).

Figura 1. Ocorrência das espécies de mamíferos de médio e grande porte registradas durante o levantamento realizado entre os meses de novembro de 2016 e julho de 2017 com armadilhas fotográficas representadas por gráficos.

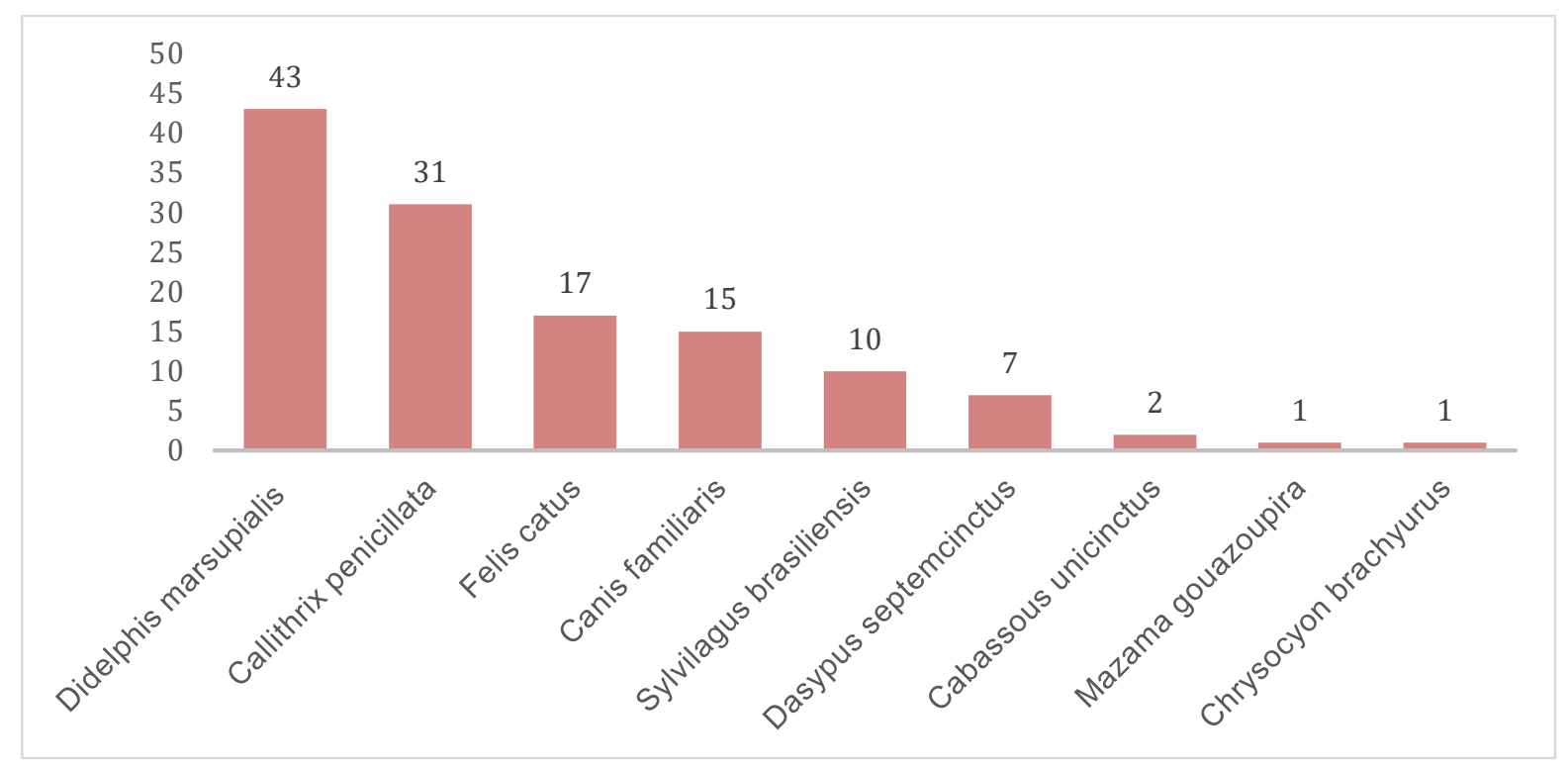

A despeito do estudo ter sido conduzido em um fragmento de cerrado obtevese registros de dois mamíferos de grande porte como o Mazama gouazoupira e Chrysocyon brachyurus (Figura 1), demonstrando que apesar da grande exigência ecológica, essas espécies pode persistir mesmo em ambientes fragmentados, uma vez que estes adaptam-se à oferta de alimentos que estão disponíveis em locais que sofreram pressões antrópicas (JUAREZ \& MARINHO-FILHO, 2002). A presença da espécie do Chrysocyon brachyurus que inclui em sua dieta alimentos frutíferos, ocorre também predações por tatus, marsupiais e veado-campeiro, fortalecendo a sua permanência no fragmento (CHEIDA et al., 2006). Entretanto, apesar de persistirem nesses ambientes, são ameaçados pela presença de animais domésticos, bem como a proximidade com as rodovias pavimentadas que ocasionam uma barreira no 
deslocamento dessas espécies (BAGATINI, 2006). Apesar do lobo-guará estar presente no bioma cerrado, é uma espécie classificada como "quase ameaça" pela lista vermelha da IUCN (IUCN, 2017) e "vulnerável" pela Lista Oficial das Espécies da Fauna Brasileira Ameaçadas de Extinção (MMA, 2003).

\subsection{Fitofisionomias e Uso do habitat}

Dentre as espécies fotografadas pelas armadilhas fotográficas, oitenta e oito (69\%) foram registradas em Mata de Galeria, vinte e cinco (20\%) em Campo Cerrado e quatorze (11\%) no Cerrado sensu estrito das frequências de mamíferos de médio e grande porte de acordo com a fitofisionomias amostradas (Figura 2).

Entre as fitofisionomias, a Mata de Galeria foi o que apresentou maior abundancia, onde os Callithrix penicillata possuíram uma maior densidade populacional da área de estudo, e que segundo Faria (1986), em áreas perturbadas e degradadas ocorre um aumento desta espécie.

Figura 2. Análise da fitofisionomia da Estação Experimental de Agroecologia comparadas ao número de espécies registradas por cada área amostrada.

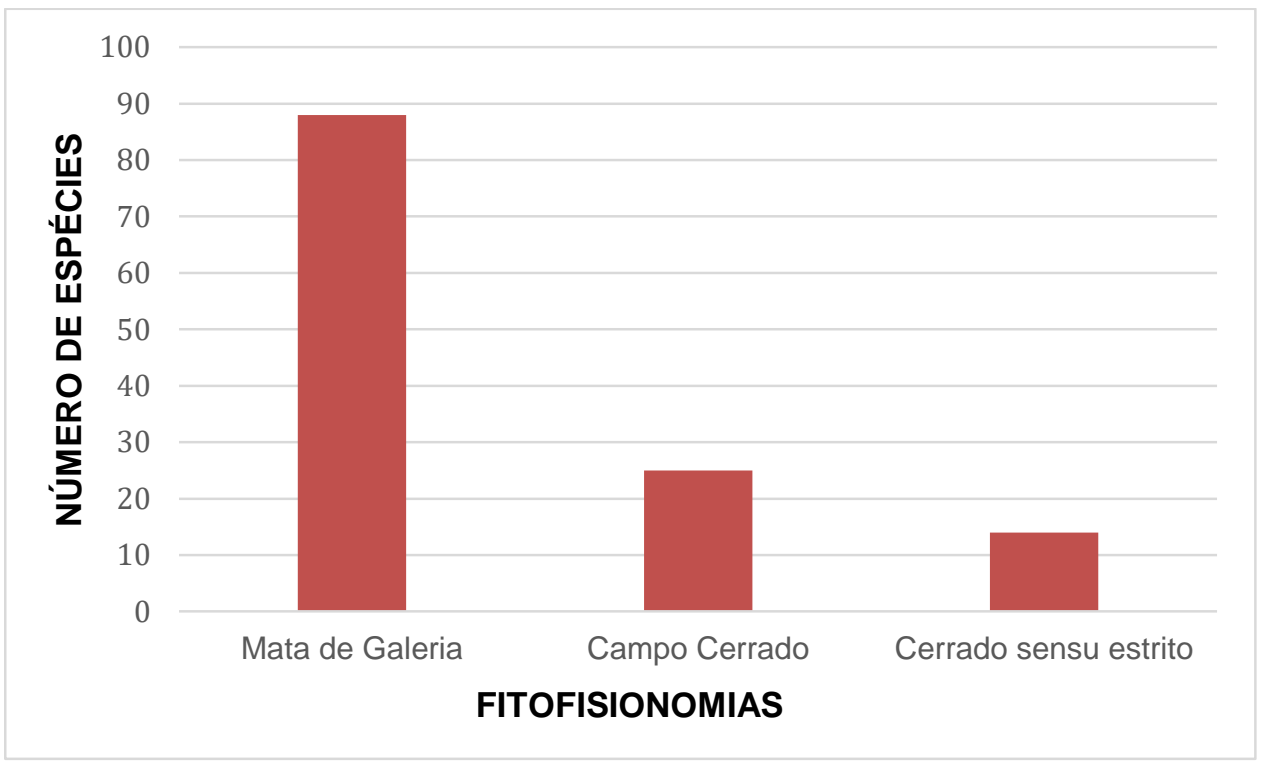

As espécies que apresentaram maior número de registros foram Didelphis marsupialis e Callithrix penicillata, que juntas representaram quase $60 \%$ dos registros fotográficos. O predomínio de Didelphis marsupialis é um indício do alto grau de perturbação da área (Tabela 2). Dados de Fonseca (1989) e Fonseca \& Robinson (1990) mostram que espécies do gênero Didelphis são generalistas de habitat e dieta 
e se adaptam muito bem a áreas onde a fauna de carnívoros esteja depauperada ou eliminada. Nessas situações, espécies do gênero Didelphis tendem a tornar-se dominante e atuam como indicativos da redução da biodiversidade de mamíferos (PASSAMANI, 2004).

\subsection{Sazonalidade}

Tabela 2. Ocorrências registradas das espécies de mamíferos de médio e grande porte durante os meses de Novembro de 2016 e Julho de 2017 na Estação Experimental de Agroecologia - Chácara Delfim, localizado na Microbacia do Córrego Urubu do Distrito Federal.

\begin{tabular}{|c|c|c|c|c|c|c|c|c|c|c|c|}
\hline Espécies & Jan & Fev & Mar & Abr & Mai & Jun & Jul & Ago Set & Out & Nov & Dez \\
\hline Cabassous unicinctus & - & - & & & & & & & & & \\
\hline Dasypus septemcinctus & - & - & - & & & & - & - & & & \\
\hline Didelphis marsupialis & - & - & - & - & - & - & - & - & & & - \\
\hline Callithrix penicillata & - & - & - & & & & & & & & \\
\hline Mazama gouazoupira & & & - & & & & & & & & \\
\hline Chrysocyon brachyurus & & & & & & & - & & & & \\
\hline
\end{tabular}

No que se refere à abundancia, quando comparadas as estações do ano, ocorre diferenças populacionais significativas que podem estar relacionadas à variações sazonais de recursos alimentares, induzindo inúmeras espécies a utilizarem diferentes períodos e locais do fragmento. Entretanto, as espécies domésticas ocorreram durante todo o período de amostragem no local do experimento, no qual representou mais de $25 \%$ dos registros fotográficos.

De forma geral, fragmentos florestais mantêm números reduzidos de espécies se comparados a trechos contínuos, pois constituem um recorte da vegetação contínua que, sobretudo em sistemas tropicais, são heterogêneas e com muitas espécies distribuídas em microhabitats dentro do ambiente florestal. Adicionalmente, fragmentos florestais possuem populações pequenas e mais suscetíveis à extinção local, além de possuir grande extensão de borda exposta a outros ambientes onde as condições ambientais resultam em alterações na estrutura da vegetação, e na composição e dinâmica da biota (LAURANCE, 2008). 


\section{Considerações Finais}

A presença de animais doméstico (Canis familiaris e Felis catus) representam uma ameaça potencial à fauna silvestre, uma vez que estes são competidores diretos por recursos alimentares, possuem a possibilidade de transmissões de doenças e alteram a densidade populacional do local. Os resultados sugerem que, a localização do fragmento, contribui para o isolamento e a restrição dos mamíferos de médio e grande porte, ocasionando implicações diretas a mastofauna.

A implementação de trabalhos de educação ambiental envolvendo as comunidades locais devem ser introduzidos como forma de minimizar os distúrbios causados pela expansão das atividades humanas. Além da realização de um inventário que manifesta a ocorrência desses mamíferos no fragmento. Com o propósito de desenvolver estratégias de conservação e conscientização do bioma Cerrado.

Recomendam-se novos estudos de longa duração com mamíferos na microbacia do Córrego do Urubu, visando monitorar flutuações populacionais e taxas de extinção e, ou, incremento de espécies. 


\section{Referências}

ALVES, L.C.P.S.; ANDRIOLO, A. 2005. Camera traps use on the mastofaunal survey of Araras Biological Reserve, IEF-RJ. Revista Brasileira de Zoociências 7 (2): 231 246.

AQUINO, F.G.; MIRANDA, H.B.M. 2008. Consequências ambientais da fragmentação de habitats no Cerrado. In Cerrado: ecologia e flora. (SANO, S.M.; ALMEIDA, S.P., eds.). Embrapa-CPAC, Planaltina, p.385-398.

BAGATINI, TATHIANA. Evolução dos índices de atropelamento de vertebrados silvestres nas rodovias do entorno da estação ecológica águas emendadas, DF, Brasil, e eficácia de medidas mitigadoras.74f. Programa de Pós-Graduação em ecologia - UnB, Brasília, 2006.

BECKER, M.; DALPONTE, J. C. Rastros de Mamíferos Silvestres Brasileiros. Um Guia de Campo. Ed. Technical Books, 2013. 166p.

BECK-KING, H.; HELVERSEN, O.V. 1999. Home range, population density, and food resources of Agouti paca (Rodentia: Agoutidae) in Costa Rica: a study using alternative methods. Biotropica 31(4):675-685.

CÂNDIDO JR, J. F. The edge effect in a forest bird community in Rio Claro, São Paulo State, Brazil. Ararajuba, v.8, n.1, p.9-16, 2000.

CARRILLO, E.; WONG, G.; CUARÓN, A.D. 2000. Monitoring mammal population in Costa Rican protected areas under different hunting restrictions. Conservation Biology 24 (6): 1580 - 91. Doi: 10.1111/j.1523-1739.2000.99103.x

COSTA, L. P. et al. Mammal conservation in Brazil. Conservation Biology, v.19, n.3, p.672-679, 2005.

CHAZDON, R.L.; HARVEY, C.A.; KOMAR, O.; GRIFFITH, D.M.; FERGUSON, B.G.; MARTÍNEZ-RAMOS, M.;MORALES, H.; NIGH, R. L.; SOTO-PINTO, M.V. B.; PHILPOTT, S.M. 2009. Beyond reserves: a research agenda for conserving biodiversity in human modified tropical landscapes. Biotropica 41: 142 153.http://dx.doi.org/10.1111/j.1744-7429.2008.00471.x

CHEIDA, C.C., E. Nakano-Oliveira, R. Fusco-Costa, F. Rocha-Mendes e J. Quadros. 2006. Ordem Carnívora, p.231-275. In: N.R. Reis, A.L. Perachi, W.A. Pedro e I.P. Lima (ed.). Mamíferos do Brasil. Londrina: UEL, 2006. p. 243-251.

CHIARELLO, A. G. Effects of fragmentation of the Atlantic forest on mammal communities in southeastern Brazil. Biological Conservation, v.89, n.1, p.71-82, 1999.

CROOKS, K.R.; SOULÉ, M.E. 1999. Mesopredator release and avifaunal extinctions in a fragmented system. Nature 400: 563 - 566. Doi:10.1038/23028.

CULLEN JR, L.; BODMER, R. E.; PÁDUA, C. V. Effects of hunting in habitat fragments of the Atlantic Forest, Brazil. Biological Conservation, v.95, n.1, p.49-56, 2000. 
CULLEN JR, L.; BODMER, R. E.; PÁDUA, C. V. Ecological consequences of hunting in Atlantic Forest patches, São Paulo, Brazil. Oryx, v.35, p.137-144, 2001.

CULLEN JÚNIOR, L.; RUDRAN, R.; VALLADARES-PADUA, C. (Orgs.). Métodos de estudos em biologia da conservação e manejo da vida silvestre. Curitiba: Universidade Federal do Paraná, 2003. p.181-201.

DeMATIA, E.A.; Curran, L.M.; RATHCKE, B.J. 2004. Effects of small rodents and large mammals on Neotropical seeds. Ecology 85(8): 2161 - 2170. http://dx.doi.org/10.1890/03-0254

DIRZO, R.; MIRANDA, A. 1990. Contemporary Neotropical defaunation and the forest structure, function, and diversity - a sequel to John Terborgh. Conservation Biology 4: 444 - 447. Doi: 10.1111/j.1523-1739.1990.tb00320.x

FAHRIG, L. Effects of habitat fragmentation on biodiversity. Annual Reviews in Ecology, Evolution and Systematics, v.34, p.487- 515, 2003.

FUTUYMA, D.J. 1997. Biologia Evolutiva. Segunda edição, Sociedade Brasileira da Genética/CNPq, Ribeirão Preto,SP.

FONSECA, G.A.B.; ROBINSON, J.G. 1990. Forest size and structure: competitive and predatory effects on small mammal communities. Biological Conservation 53: 265 294. Doi: 10.1016/0006-3207(90)90097-9.

FONSECA GAB e KH REDFORD. The mammals of IBGE's Ecological Reserve, Brasília, and an analysis of the role of gallery forests in increasing diversity. Revista Brasileira de Biologia 44:517-523. 1984.

FONSECA, G.A.B. \& ROBINSON, J.G. Forest size and structure: competitive and predatory effects on small mammals communities. Biol. Conserv. 53:265-294. 1990.

FARIA, D. S. 1986. O Estudo de campo do "Mico-Estrela" Callithrix penicillata do Planalto Central Brasileiro. In: Etologia de Animais e de Homens. César Ades (ed), Edicon/EDUSP - São Paulo,1989. P.109-121.

GARDNER, T.A.; BARLOW, J.; CHAZDON, R.; EWERS, R.M.; HARVEY, C.A.; PERES, C.A.; SODHI, N.S. 2009.Prospects for tropical forest biodiversity in a humanmodified world. Ecology Letters 12: 561 - 582.http://dx.doi.org/10.1111/j.14610248.2009.01294.x

HENRY O. 1999. Frugivory and the importance of seeds in the diet of the orangerumped agouti (Dasyprocta leporina) in French Guiana. Journal of Tropical Ecology 15:291-300.

IUCN, The IUCN Red List of Threatened Species. <http://www.iucnredlist.org/>. Acesso em 29/07/2017. 2017-1.

JUAREZ, K.M; MARINHO-FILHO, J. Diet, habitat use, and home ranges of sympatric canids in central Brazil. Jornal of mammalogy, v. 83, n.4, p. 925-933, nov. 2002. 
KASPER, C.B.; FELDENS, M.J.; MAZIM, F.D.; SCHNEIDER, A.; CADEMARTORI, C.V.; GRILLO, H.C.Z. 2007. Mamíferos do Vale do Taquari, região central do Rio Grande do Sul. Biociências (On-line), 15.

LAURANCE, W.F. 2008. Theory meets reality: How habitat fragmentation research has transcended island. biogeographic theory. Biological Conservation 141: 1731 1744. Doi: 10.1016/j.biocon.2008.05.011.

LIMA, M. G.M. Mamíferos de médio e grande porte do parque nacional das nascentes do Rio Parnaíba, Brasil. 2009. 160 f. Tese (Mestrado) - Programa de Pós-Graduação em Zoologia da Universidade Federal do Pará, 2009.

MARINHO-FILHO, J. S.; RODRIGUES, F.H.G.; JUAREZ, K. M. 2002. The Cerrado mammals: diversity, ecology and natural history, 266-284, em: The Cerrados of Brazil: Ecology and Natural History of Mastozoología Neotropical,16(2):355-364, Mendoza, 2009.

MINISTÉRIO DO MEIO AMBIENTE - MMA. Espécies da fauna brasileira ameaçadas de extinção. Instrução Normativa $n^{\circ} 3$, de 27 de maio de 2003, publicada no Diário Oficial da União n 101, de 28 de maio de 2003. Seção 1. p. 88-97.

NASCIMENTO, et al. 2004. Período de atividade de alguns vertebrados do Pantanal, estimado por fotografia remota. IV Simpósio sobre Recursos Naturais e Sócioeconomicos do Pantanal, Corumbá/MS.

PALOMARES F, P GAONA, P FERRERAS e M DELIBES. 1995. Positive effects on game species of top predators by controlling smaller predator populations: an example with Lynx, Mongooses and Rabbits. Conservation Biology 9:295-305.

PARDINI, R. et al. Levantamento rápido de mamíferos terrestres de médio e grande porte. In: CULLEN JÚNIOR, L.; RUDRAN, R.; VALLADARES-PADUA, C. (Orgs). Métodos de estudos em biologia da conservação e manejo da vida silvestre. Curitiba: Universidade Federal do Paraná, 2003. p. 19-21.

PASSAMANI, M.; DALMASCHIO, J.; LOPES, S.A.; Mamiferos não-voadores em áreas com predomínio de Mata Atlantica da Amarco Mineração S.A, município de Anchieta, Espirito Santo. Biotemas. Santa Catarina, 31 de agosto de 2004.

PERES, C. A. Synergistic effects of subsistence hunting and habitat fragmentation on Amazonian Forest vertebrates. Conservation Biology, v.5, n.6, p.1490-1505, 2001.

ROCHA-MENDES, F. et al. Mamíferos do município de Fênix, Paraná, Brasil: etnozoologia e conservação. Revista Brasileira de Zoologia, v.22, n.4, p.991-1002, 2005.

SANTOS-FILHO, M.; SILVA, M.N.F. 2002. Uso de hábitats por mamíferos em área de Cerrado do Brasil Central: um estudo com armadilhas fotográficas. Revista Brasileira de Zoociências 4:57-73. 
SECADES, C.; O?CONNOR, B.; BROWN, C.; WALPOLE, M. 2014. Earth observation for biodiversity monitoring: a review of current approaches and future opportunities for 35 tracking progress towards the Aichi biodiversity targets (Secretariat of the Convention on Biological Diversity, Montreal, Canada, Technical Series No. 72).

SILVEIRA, L.; JÁCOMOA, A.T.A.; DINIZ-FILHO, J.A.F. 2003. Camera trap, line transect census and track surveys: a comparative evaluation. Biological Conservation 114: 351 - 355. Doi: 10.1016/S0006-3207(03)00063-6.

TERBORGH, J. 1988. The big things that run the world $\square \square$ a sequel to E.O. Wilson. Conservation Biology 2: 402 - 403.Doi: 10.1111/j.1523-1739.1988.tb00207.x

TERBORGH, J.; LOPEZ, L.; NUÑEZ V. P.; RAO, M.; SHAHABUDDIN, G.; ORIHUELA, G.; RIVEROS, M.; ASCANIO,R.; ADLER, G.H.;. LAMBERT, T.D.; BALBAS, L. 2001. Ecological meltdown in predator-free forest fragments. Science 294: 1923 - 1926. Doi: 10.1126/science.1064397.

TROLLE, M. 2003a. Mammal survey in the Rio Jauaperí region, Rio Negro Basin, the Amazon, Brazil. Mammalia 67 (1): 75-83.

SRBEK-ARAUJO, A.C.; CHIARELLO, A.G. 2005. Is camera trapping an efficient method for surveying mammals in Neotropical forests? A case study in southeastern Brazil. Journal of Tropical Ecology 21: 121 125.http://dx.doi.org/10.1017/S0266467404001956.

WILCOX, B.A.; MURPHY, D.D. 1985. Conservation strategy: the effects of fragmentation on extinction. The American Naturalist 125: 879 - 887. 